\title{
Article \\ Numerical Solution of Linear Volterra Integral Equation Systems of Second Kind by Radial Basis Functions
}

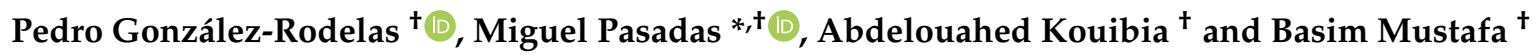 \\ Department of Applied Mathematics, Granada University, 18071 Granada, Spain; prodelas@ugr.es (P.G.-R.); \\ kouibia@ugr.es (A.K.); bmustafa@correo.ugr.es (B.M.) \\ * Correspondence: mpasadas@ugr.es \\ † These authors contributed equally to this work.
}

\begin{abstract}
In this paper we propose an approximation method for solving second kind Volterra integral equation systems by radial basis functions. It is based on the minimization of a suitable functional in a discrete space generated by compactly supported radial basis functions of Wendland type. We prove two convergence results, and we highlight this because most recent published papers in the literature do not include any. We present some numerical examples in order to show and justify the validity of the proposed method. Our proposed technique gives an acceptable accuracy with small use of the data, resulting also in a low computational cost.
\end{abstract}

Keywords: Volterra integral equations system; radial basis functions; variational methods

check for updates

Citation: González-Rodelas, P.;

Pasadas, M.; Kouibia, A.; Mustafa, B. Numerical Solution of Linear Volterra Integral Equation Systems of Second Kind by Radial Basis Functions. Mathematics 2022, 10, 223. https://doi.org/10.3390/ math10020223

Academic Editors: Sara Remogna, Domingo Barrera, María José Ibáñez and Simeon Reich

Received: 30 November 2021

Accepted: 4 January 2022

Published: 12 January 2022

Publisher's Note: MDPI stays neutral with regard to jurisdictional claims in published maps and institutional affiliations.

Copyright: (c) 2022 by the authors. Licensee MDPI, Basel, Switzerland. This article is an open access article distributed under the terms and conditions of the Creative Commons Attribution (CC BY) license (https:// creativecommons.org/licenses/by/ $4.0 /)$.

\section{Introduction}

A considerable large amount of research literature and books on the theory and applications of Volterra's integral equations have emerged over many decades since the apparition of Volterra's book "Leçons sur les équations intégrales et intégro-différentielles" [1] in 1913.

The applications include elasticity, plasticity, semi-conductors, scattering theory, seismology, heat and mass conduction or transfer, metallurgy, fluid flow dynamics, chemical reactions, population dynamics, and oscillation theory, among many others (see for example [2]). Other important references more related with the numerics of this type of equation are $[3,4]$.

In fact, Volterra integral equations (VIEs) appear naturally when we try to transform an initial value problem into integral form, so that the solution of this integral equation is usually much easier to obtain than the original initial value problem. In the same way, some nonlinear Volterra integral equations are equivalent to an initial-value problem for a system of ordinary differential equations (ODEs). So, some authors (like for example [5]) have sought to exploit this connection for the numerical solution of the integral equations as well, since very effective ODE codes are widely available.

Volterra integral equations arise in many usual applications of technology, engineering and science in general: as in population dynamics, the spread of epidemics, some Dirichlet problems in potential theory, electrostatics, mathematical modeling of radioactive equilibrium, the particle transport problems of astrophysics and reactor theory, radiative energy and/or heat transfer problems, other general heat transfer problems, oscillation of strings and membranes, the problem of momentum representation in quantum mechanics, etc. However, many other complex problems of mathematics, chemistry, biology, astrophysics and mechanics, can be expressed in the terms of Volterra integral equations. Moreover, some practical problems, where impulses arise naturally (like in population dynamics or many biological applications) or are caused by some control system (like electric circuit problems and simulations of semiconductor devices) can be modeled by a differential equation, an integral equation, an integro-differential equation, or a system of these equations all combined. 
The systems of integral and/or integro-differential equations are usually difficult to solve analytically, in particular systems of Volterra integral non-linear equations or with variable coefficients; so a numerical method is often needed. In such cases, it is required to approximate the solutions; and many different numerical techniques have been developed and presented during decades of research, with appropriate combinations of numerical integration and interpolation procedures (see the references [3,6], among others). In order to approximate numerically the solution of general integral equations, the predominant technique have been the use of some kind of piecewise constant basis functions (PCBFs) (see for example [7], among many others); Chebyshev polynomial ([8] and others). However, after a long period of time many other techniques have attracted much attention recently; like wavelets theory, started with the introduction of Haar function in 1910, and from 1990's (see [9]) also many wavelet type methods have been applied for solving integral equations. Haar wavelets, despite its relative simplicity, have many valued properties: as its compact support and orthogonality properties. So they can be used for the solution of differential and integro-differential equations related with signal and image processing, for example. They have been also used to solve linear and nonlinear integral equations by Aziz et. al. [10], Babolian et. al. [11], Lepik [12], Maleknejad et al. [13], Farshid Mirzaae [14], among others. More recently, several numerical methods based on different triangular type and delta orthogonal functions were designed for approximating the solution of integral and/or integro-differential Volterra equations (see for example [15-17], and the references therein). All these publications have demonstrated and revealed that these techniques based on PCBF and wavelets are effective to obtain the solution of such integral equations.

Particularly, systems of linear integral equations, and their exact or approximate solutions, are of great importance in science and engineering. There are several numerical methods for solving systems of linear Volterra integral equations of the second kind, and they have been often solved by classical numerical and analytical methods: such as Galerkin and Finite Element methods, collocation and spectral methods, Taylor or Power series and expansion methods, transforming the equations into a linear or nonlinear system of algebraic equations, and so on. However, new methods also have been applied to solve them, like the homotopy perturbation method [18], Adomian decomposition method (and many others) [19], use of Legendre wavelets [20] or hybrid Legendre and block pulse functions [21], Chebyshev polynomials [22,23], etc. Berenguer et al. [24,25] have solved them with the aid of a combination of analytical methods and bi-orthogonal systems in Banach spaces, Sahn et al. [26] have used Bessel polynomials method, Malnekad et al. [15] have employed delta basis functions (DBFs), Balakumar et al. [27] have applied the blockpulse functions method, Li-Hong et al. [28] have applied reproducing kernel method. Furthermore, there are also expansion methods for integral equations such as El-gendi's and Wolfe's methods (see for example [29]). Additionally, the approximate solutions of systems of integral equations that usually appear in problems of physics, biology and engineering are based on numerical integration methods: such as Euler-Chebyshev or Runge-Kutta methods (see for example [30]).

Concerning many other possible techniques to solve these types of integral equations, Draidi and Qatanani [31] implemented a product Nystrom and sinc-collocation methods to solve Volterra integral equations with Carleman kernel; also Issa, Qatanani and Daraghmeh [32] used a Taylor expansion and the variational iteration methods to give an approximate solution of Volterra integral equations of the second kind. Aggarwal et al. [33] and Chauhan [34] used different integral transformations for obtaining the solutions of VIEs of second kind. Mahgoub [35] solved constant coefficient linear differential equations by defining the called Sawi transformation, but many other authors exploited this idea, or other appropriate transforms, to deal with these types of integral or integro-differential equations.

Next, we are going to cite the most recent references from the last 3 or 4 years. In [36] the authors present an approximation solution of system of Volterra integral equations of second kind in an analytical way, using an Adomian decomposition method in Mathematica. In [37] the authors propose a numerical algorithm based on Monte Carlo method for 
approximating solutions of the system of Volterra integral equations. In [38] the authors develop a numerical technique for the solution of 2D Volterra integral equations based on a discretization method by using two-dimensional Bernstein's approximations. In [39] the authors discussed the solution of linear Volterra integral equations of second kind using Mohand transform. In [40] the authors propose Bernstein polynomials to present effective solution for the second kind linear Volterra integral equations with delay. In [41] the author presents a method to solve numerically Volterra integral equations of the first kind with separable kernels.

In this work, we will present some specific variational methods adopted to study and approximate systems of linear Volterra integral equations with the aid of Radial Basis Functions (RBFs) of Wendland type. Wendland functions are compactly supported radial basis functions, which makes calculations with them quite simple. However, the general Wendland family of functions are defined recursively, and to determine the actual functions to use in any software implementation many calculations had to be done by hand or with the aid of some symbolic software (see for example [42]). There are for the moment just a few articles dealing with this type of techniques, like for example [43-47]; so we think there is still a lot to investigate in this regard.

Our goal in this work is to devise an appropriate approach procedure that is capable of solving this type of problem in a precise and efficient way. We consider then the linear Volterra equations system of the second kind as follows (see for example [1]):

$$
x(t)=f(t)+\int_{0}^{t} k(t, s) x(s) d s, \quad 0 \leq s \leq t \leq 1,
$$

where

$$
\begin{aligned}
& x(t)=\left(x_{1}(t), \ldots, x_{n}(t)\right)^{\top}, \\
& f(t)=\left(f_{1}(t), \ldots, f_{n}(t)\right)^{\top}, \\
& \boldsymbol{k}(t, s)=\left(k_{i j}(t, s)\right)_{1 \leq i, j \leq n} .
\end{aligned}
$$

We assume that (1) has a unique continuous solution for appropriate functions $f$. In any case, the equations system (1) can be re-written in operator form as an equation of second kind

$$
f=(I-K) x,
$$

where $K$ is an integral operator and $I$ denotes the identity operator. It is usual to impose certain assumptions on compactness on the operator $K$ (see [48], Section 2.8.1) in order to establish the existence and uniqueness of the solution of (1), that we will assume throughout the work.

Moreover, in [49] the authors proposed another method to solve second kind Fredholm integral equation systems, but the discrete functional space chosen in that article has been the space of spline functions. While at first glance it might seem that both works are similar, especially in the way they are presented, the two methods are totally different, not only be the fact that the discretization spaces are different (so we have adapted the notations accordingly), while the proofs (except the very preliminary ones, that can be also adapted), above all the proofs of the convergence results, are completely different, due to their greater complexity.

The outline of the paper is as follows. In Section 2 we briefly recall some notations and preliminaries. Section 3 is devoted to establish the discretization space as a radial basis functions space. The formulation of the minimization problem is realized in Section 4 and two equivalent variational problems are given. Section 5 is devoted to prove two convergence results. Section 6 deals with the description of the computation algorithm of the discrete problem solution. In Section 7 we present some numerical experiments and finally, in Section 8 we establish the conclusions of the work. 


\section{Notations and Preliminaries}

Let $\mathbb{R}_{0}^{+}=\{x \in \mathbb{R}: x \geq 0\}$; and for $n \geq 1$, we denote by $\langle\cdot\rangle_{n}$ and $\langle\cdot, \cdot\rangle_{n}$ the Euclidean norm and the inner product in $\mathbb{R}^{n}$.

On the other hand, for $m \geq 1$, we designate by $H^{m}\left((0,1) ; \mathbb{R}^{n}\right)$ the Sobolev space of order $m$ of (classes of) functions $u \in L^{2}\left((0,1) ; \mathbb{R}^{n}\right)$ together with all $j$-th derivative functions $\boldsymbol{u}^{(j)}$ of order $j \leq m$, in the sense of distributions. This space is equipped with

- $\quad$ the semi-inner products, for any $\boldsymbol{u}, \boldsymbol{v} \in H^{m}\left((0,1) ; \mathbb{R}^{n}\right)$,

$$
(\boldsymbol{u}, \boldsymbol{v})_{j}=\int_{0}^{1}\left\langle\boldsymbol{u}^{(j)}(t), \boldsymbol{v}^{(j)}(t)\right\rangle_{n} d t, \quad 0 \leq j \leq m,
$$

- $\quad$ the corresponding semi-norms $|\boldsymbol{u}|_{j}=(\boldsymbol{u}, \boldsymbol{u})_{j}^{\frac{1}{2}}$, for $0 \leq j \leq m$,

- $\quad$ the inner product $((\boldsymbol{u}, \boldsymbol{v}))_{m}=\sum_{0}^{m}(\boldsymbol{u}, \boldsymbol{v})_{j}$,

- $\quad$ and the corresponding norm $\|\boldsymbol{u}\|_{m}=((\boldsymbol{u}, \boldsymbol{u}))_{m}^{\frac{1}{2}}$.

For any $1 \leq i \leq n$, let $\boldsymbol{k}_{i}$ be a given function of the Sobolev vectorial functions space $H^{m}\left((0,1) \times(0,1) ; \mathbb{R}^{n}\right)$ and consider the matrix valued function

$$
\boldsymbol{k}(t, s)=\left(\boldsymbol{k}_{i}(t, s)\right)_{1 \leq i \leq n} \in H^{m}\left((0,1) \times(0,1) ; \mathbb{R}^{n, n}\right),
$$

together with the associated integral operator

$$
K \boldsymbol{u}(t)=\left(\int_{0}^{t}\left\langle\boldsymbol{k}_{i}(t, s), \boldsymbol{u}(s)\right\rangle_{n} d s\right)_{1 \leq i \leq n}, \quad t \in(0,1), \forall \boldsymbol{u} \in H^{m}\left((0,1) ; \mathbb{R}^{n}\right) .
$$

Let $\mathbb{R}^{n, p}$ be the space of real matrices of $n$ lines and $p$ columns, equipped with the inner product

$$
\langle A, B\rangle_{n, p}=\sum_{i=1}^{n} \sum_{j=1}^{p} a_{i j} b_{i j}, \quad \forall A=\left(a_{i j}\right)_{\substack{1 \leq i \leq n \\ 1 \leq j \leq p}}, B=\left(b_{i j}\right)_{\substack{1 \leq i \leq n \\ 1 \leq j \leq p}} \in \mathbb{R}^{n, p},
$$

and the corresponding norm $\langle A\rangle_{n, p}=\langle A, A\rangle_{n, p}^{\frac{1}{2}}$.

\section{Discretization Space}

For the remainder of the work, we are going to consider a space of finite dimension, where we will formulate and solve a discrete approximation problem. The discrete functional space we have chosen is the radial basis functions space with compact support, namely the radial basis function space generated by the Wendland functions (see [50]).

Definition 1. Given a continuous function $\phi: \mathbb{R}_{0}^{+} \rightarrow \mathbb{R}$, a subset $\Omega \subset \mathbb{R}^{d}, d \geq 1$, and a point $\xi \in \Omega$, the radial function defined on $\Omega$ from the function $\phi$ with center $\xi$ is the continuous function $\Phi_{\xi}: \Omega \rightarrow R$ given by

$$
\boldsymbol{\Phi}_{\boldsymbol{\xi}}(\boldsymbol{x})=\phi\left(\langle\boldsymbol{x}-\boldsymbol{\xi}\rangle_{d}\right) .
$$

Then $\boldsymbol{\Phi}_{\xi}$ only depends of the distance to $\boldsymbol{\xi}$.

Definition 2. Given a centers set $\Xi=\left\{\xi_{1}, \ldots, \xi_{N}\right\}$ the linear space generated by the functions

$$
\left\{\phi\left(\left\langle\cdot-\xi_{1}\right\rangle\right)_{d}, \ldots,\left\{\phi\left(\left\langle\cdot-\xi_{N}\right\rangle\right)_{d}\right\}\right.
$$

is called a radial basis functions space. 
Definition 3. For a function $\boldsymbol{u} \in C\left([0,1] ; \mathbb{R}^{n}\right)$, the radial basis function interpolating $\boldsymbol{u}$ on a set of distinct centers $\boldsymbol{T}_{N}=\left\{t_{1}, \ldots, t_{N}\right\} \subset[0,1]$ is given by

$$
\boldsymbol{s}_{\boldsymbol{u}, \boldsymbol{T}_{N}}(t)=\sum_{i=1}^{N} \boldsymbol{\alpha}_{i} \phi\left(\left|t-t_{i}\right|\right), \quad t \in[0,1],
$$

where $\phi: \mathbb{R}_{0}^{+} \rightarrow \mathbb{R}$ is a continuous function and the coefficients $\boldsymbol{\alpha}_{1}, \ldots, \boldsymbol{\alpha}_{N} \in \mathbb{R}^{n}$ are determined by the interpolation conditions

$$
\boldsymbol{s}_{\boldsymbol{u}, \boldsymbol{T}_{N}}\left(t_{i}\right)=\boldsymbol{u}\left(t_{i}\right), \quad 1 \leq i \leq N .
$$

In [50] H. Wendland introduced a family of compactly supported radial basis functions in the following way: let the operator $\mathcal{I}$ and its inverse $\mathcal{D}$ for $r \geq 0$ be given by

$$
\begin{aligned}
& (\mathcal{I} \phi)(r)=\int_{r}^{\infty} t \phi(t) d t, \\
& (\mathcal{D} \phi)(r)=-\frac{1}{r} \phi^{\prime}(r),
\end{aligned}
$$

for any differentiable function $\phi: \mathbb{R}_{0}^{+} \rightarrow \mathbb{R}$.

Given the truncated power function $\phi_{\ell}(r)=(1-r)_{+}^{\ell}$, we define

$$
\phi_{d, k}=\mathcal{I}^{k} \phi_{\left\lfloor\frac{d}{2}\right\rfloor+k+1^{\prime}}
$$

where $\lfloor x\rfloor$ denotes the largest integer less than or equal to $x$.

Theorem 1. ([50], Theorem 1.2) The functions $\phi_{d, k}$ induce positive definite functions on $\mathbb{R}^{d}$ of the form

$$
\phi_{d, k}(r)= \begin{cases}p_{d, k}(r), & 0 \leq r \leq 1, \\ 0, & r>1,\end{cases}
$$

with a univariate polynomial $p_{d, k}$ of degree $\left\lfloor\frac{d}{2}\right\rfloor+3 k+1$. They possess continuous derivatives up to order $2 k$, and they are of minimal degree for a given constant factor, uniquely determined by this setting.

Thus, these functions are the natural candidates for interpolation by compactly supported radial basis functions, and they are called the Wendland's functions.

For the remainder of the work we suppose $0 \leq k \leq N-1$, and we take $\phi=\phi_{1, k}$ in Definition 3.

Table 1 shows the Wendland functions $\phi_{1, k}$ for $k=0,1,2$, and its continuity order.

Table 1. The Wendland functions $\phi_{1, k}$ for $k=0,1,2$ and its continuity order.

\begin{tabular}{clc}
\hline $\boldsymbol{k}$ & Wendland Function & Continuity Order \\
\hline$k=0$ & $\phi_{1,0}(r)=(1-r)_{+}$ & $C^{0}$ \\
$k=1$ & $\phi_{1,1}(r) \doteq(1-r)_{+}^{3}(3 r+1)$ & $C^{2}$ \\
$k=2$ & $\phi_{1,2}(r) \doteq(1-r)_{+}^{5}\left(8 r^{2}+5 r+1\right)$ & $C^{4}$ \\
\hline
\end{tabular}

Let

$$
h=\sup _{t \in[0,1]} \min _{1 \leq i \leq N}\left|t-t_{i}\right| .
$$

From ([50], Theorem 2.1) we can affirm that $\phi_{1, k} \in C^{2 k}([0,1])$ and the corresponding native space is $H^{k+1}([0,1])$. Finally, from ([50], Theorem 2.1) and ([51], Theorem 4.1) we conclude that there exists $C>0$ such that 


$$
\left\|\boldsymbol{u}-\boldsymbol{s}_{\boldsymbol{u}, \boldsymbol{T}_{N}}\right\|_{L_{\infty}\left((0,1) ; \mathbb{R}^{n}\right)} \leq C\|\boldsymbol{u}\|_{k+1} h^{k+\frac{1}{2}}, \quad \forall \boldsymbol{u} \in H^{k+1}\left([0,1] ; \mathbb{R}^{n}\right)
$$

and

$$
\left|\boldsymbol{u}-\boldsymbol{s}_{\boldsymbol{u}, \boldsymbol{T}_{N}}\right|_{j} \leq C h^{k+1-j}\|\boldsymbol{u}\|_{k+1}, \quad 0 \leq j \leq k+1, \quad \forall \boldsymbol{u} \in H^{k+1}\left([0,1] ; \mathbb{R}^{n}\right) .
$$

Let $S_{N}$ be the space of the restrictions of functions on $[0,1]$ of the functional space generated by the radial basis functions $\left\{\phi_{1, k}\left(\left|\cdot-t_{1}\right|\right), \ldots, \phi_{1, k}\left(\left|\cdot-t_{N}\right|\right)\right\}$ and $S_{N}=\left(S_{N}\right)^{n}$. Then $S_{N} \subset H^{k+1}\left((0,1) ; \mathbb{R}^{n}\right) \cap C^{2 k}\left([0,1] ; \mathbb{R}^{n}\right)$.

\section{Formulation of the Problem}

We can define the operator $\rho: H^{k+1}\left((0,1) ; \mathbb{R}^{n}\right) \rightarrow \mathbb{R}^{N, n}$ given by

$$
\rho \boldsymbol{v}=\left((I-K) v\left(t_{i}\right)\right)_{1 \leq i \leq N} .
$$

Let assume that $f \in H^{k+1}\left((0,1) ; \mathbb{R}^{n}\right)$ and consider the affine variety $\boldsymbol{H}_{N}=\left\{\boldsymbol{u} \in \boldsymbol{S}_{N}\right.$ : $\left.\rho \boldsymbol{u}=\left(\boldsymbol{f}\left(t_{i}\right)\right)_{1 \leq i \leq N}\right\}$ and the linear subspace $\boldsymbol{H}_{N}^{0}=\left\{\boldsymbol{u} \in \boldsymbol{S}_{N}: \rho \boldsymbol{u}=\mathbf{0} \in \mathbb{R}^{N, n}\right\}$.

Proposition 1. The set $\boldsymbol{H}_{N}$ is a nonempty closed bounded convex subset of $\boldsymbol{S}_{N}$. Moreover it is an affine variety associated with the linear subspace $\boldsymbol{H}_{\mathrm{N}}^{0}$.

Proof. By adapting the notations, as in the proof of Proposition 4.1 of [49].

Lemma 1. The application $\ll \cdot, \cdot \gg: H^{k+1}\left((0,1) ; \mathbb{R}^{n}\right) \times H^{k+1}\left((0,1) ; \mathbb{R}^{n}\right) \rightarrow \mathbb{R}$ defined by

$$
\ll \boldsymbol{u}, \boldsymbol{v} \gg=\langle\rho \boldsymbol{u}, \rho \boldsymbol{v}\rangle_{N, n}+((I-K) \boldsymbol{u},(I-K) \boldsymbol{v})_{k+1}
$$

is an inner product on $H^{k+1}\left((0,1) ; \mathbb{R}^{n}\right)$ and its associated norm, given by $[[\boldsymbol{u}]]=\ll \boldsymbol{u}, \boldsymbol{u} \gg^{\frac{1}{2}}$, is equivalent to the usual Sobolev norm $\|\cdot\|_{k+1}$.

Proof. By adapting the notations as in the proof of Lemma 4.2 of [49] and using ([48], Theorem 7.3.12) the proof can be obtained.

Definition 4. We say that $\boldsymbol{u}_{N} \in \boldsymbol{H}_{N}$ is an approximating radial basis function relative to $\boldsymbol{T}_{N}, \rho$ and $f$ if $\boldsymbol{u}_{N}$ is a solution of the following minimization problem:

$$
\text { Find } \boldsymbol{u}_{N} \in \boldsymbol{H}_{N} \text { such that } \forall \boldsymbol{v} \in \boldsymbol{H}_{N}, \quad J\left(\boldsymbol{u}_{N}\right) \leq J(\boldsymbol{v}),
$$

where $J: H^{k+1}\left((0,1) ; \mathbb{R}^{n}\right) \rightarrow \mathbb{R}$ is given by

$$
J(\boldsymbol{v})=|(I-K) \boldsymbol{v}|_{k+1}^{2} .
$$

Theorem 2. Problem (4) has a unique solution $\boldsymbol{u}_{N} \in \boldsymbol{H}_{N}$ which is the unique solution of the variational problem

$$
\forall \boldsymbol{v} \in \boldsymbol{H}_{N^{\prime}}^{0} \quad\left((I-K) \boldsymbol{u}_{N},(I-K) \boldsymbol{v}\right)_{k+1}=0 .
$$

Proof. From Proposition 1 and ([48], Theorem 3.4.3) we can deduce that there exists a unique $\boldsymbol{u}_{N} \in \boldsymbol{H}_{N}$, which is the projection of $\mathbf{0}$ on $\boldsymbol{H}_{N}$ such that

$$
\left[\left[\boldsymbol{u}_{N}\right]\right] \leq[[\boldsymbol{v}]], \forall \boldsymbol{v} \in \boldsymbol{H}_{N}
$$

and verifying

$$
\forall \boldsymbol{w} \in \boldsymbol{H}_{N}, \quad \ll-\boldsymbol{u}_{N}, \boldsymbol{w}-\boldsymbol{u}_{N} \gg \leq 0,
$$

that is

$$
\forall \boldsymbol{v} \in \boldsymbol{H}_{N^{\prime}}^{0} \quad \ll-\boldsymbol{u}_{N}, \boldsymbol{v} \gg \leq 0
$$


and, taking into account that $\boldsymbol{H}_{N}^{0}$ is a vector space, we obtain that

$$
\forall \boldsymbol{v} \in \boldsymbol{H}_{N^{\prime}}^{0} \quad \ll \boldsymbol{u}_{N}, \boldsymbol{v} \gg=0 .
$$

Therefore (5) holds. Finally, $\boldsymbol{u}_{N}$ is the unique solution of (4) since $J(\boldsymbol{v})=[[\boldsymbol{v}]]^{2}-$ $\langle\rho f\rangle_{N, n}^{2}$, for any $\boldsymbol{v} \in \boldsymbol{H}_{N}$.

Theorem 3. There exists a unique $\lambda \in \mathbb{R}^{N, n}$ such that

$$
\forall \boldsymbol{v} \in \boldsymbol{S}_{N}, \quad\left((I-K) \boldsymbol{u}_{N},(I-K) \boldsymbol{v}\right)_{k+1}+\langle\lambda, \rho \boldsymbol{v}\rangle_{N, n}=0,
$$

where $\boldsymbol{u}_{N}$ is the unique solution of (5).

Proof. For $i=1, \ldots, N$, let us consider $\varphi_{i} \in S_{N}$ the unique radial basis function determined by the interpolation conditions

$$
\varphi_{i}\left(t_{j}\right)=\delta_{i j}, \forall j=1, \ldots N .
$$

Let take $v \in S_{N}$, and we consider the function

$$
\boldsymbol{w}=\boldsymbol{v}-\sum_{i=1}^{N}(I-K) \boldsymbol{v}\left(t_{i}\right) \varphi_{i}
$$

then

$$
(I-K) \boldsymbol{w}\left(t_{j}\right)=(I-k) \boldsymbol{v}\left(t_{j}\right)-\sum_{i=1}^{N}(I-K) \boldsymbol{v}\left(t_{i}\right) \varphi_{i}\left(t_{j}\right)=0, \forall j=1, \ldots, N,
$$

that is $\rho \boldsymbol{w}=\mathbf{0} \in \mathbb{R}^{N, n}$, and in fact $\boldsymbol{w} \in \boldsymbol{H}_{N}^{0}$. Thus, from Theorem 2, we have

$$
\left((I-K) \boldsymbol{u}_{N},(I-K) \boldsymbol{w}\right)_{k+1}=0 .
$$

We notice $\Pi_{\ell}: \mathbb{R}^{n} \rightarrow \mathbb{R}$, for $\ell=1, \ldots, n$, the projection application given by $\Pi_{\ell}\left(x_{1}, \ldots, x_{n}\right)=x_{\ell}$.

Then, for $i=1, \ldots, N$, it is verified that

$$
\begin{aligned}
\left((I-K) \boldsymbol{u}_{N},(I-K) \boldsymbol{v}\left(t_{i}\right) \varphi_{i}\right)_{k+1} & =\sum_{\ell=1}^{n}\left(\Pi_{\ell}\left((I-K) \boldsymbol{u}_{N}, \Pi_{\ell}\left((I-K) \boldsymbol{v}\left(t_{i}\right) \varphi_{i}\right)\right)_{k+1}\right. \\
& =\sum_{\ell=1}^{n} \Pi_{\ell}\left((I-K) \boldsymbol{v}\left(t_{i}\right)\right)\left(\Pi_{\ell}\left((I-K) \boldsymbol{u}_{N}, \varphi_{i}\right)_{k+1} .\right.
\end{aligned}
$$

Let denote $\lambda_{i \ell}=-\left(\Pi_{\ell}\left((I-K) \boldsymbol{u}_{N}, \varphi_{i}\right)_{k+1} \in \mathbb{R}\right.$ and $\lambda=\left(\lambda_{i \ell}\right)_{\substack{1 \leq i \leq N \\ 1 \leq \ell \leq n}} \in \mathbb{R}^{N, n}$, then

$$
\begin{aligned}
& \left((I-K) \boldsymbol{u}_{N}(I-K) \boldsymbol{w}\right)_{k+1}= \\
& \left((I-K) \boldsymbol{u}_{N}(I-K) \boldsymbol{v}\right)_{k+1}-\sum_{i=1}^{N}\left((I-K) \boldsymbol{u}_{N}(I-K) \boldsymbol{v}\left(t_{i}\right) \varphi_{i}\right)_{k+1}= \\
& \left((I-K) \boldsymbol{u}_{N}(I-K) \boldsymbol{v}\right)_{k+1}+\sum_{i=1}^{N} \sum_{\ell=1}^{n} \Pi_{\ell}\left((I-K) \boldsymbol{v}\left(t_{i}\right)\right) \lambda_{i \ell}= \\
& \left((I-K) \boldsymbol{u}_{N}(I-K) \boldsymbol{v}\right)_{k+1}+\langle\lambda, \rho \boldsymbol{v}\rangle_{N, n} .
\end{aligned}
$$

From (7), we conclude that there exists $\lambda=\left(-\left(\Pi_{\ell}\left((I-K) \boldsymbol{u}_{n}\right), \varphi_{i}\right)_{k+1}\right)_{\substack{1 \leq i \leq N \\ 1 \leq \ell \leq n}} \in \mathbb{R}^{N, n}$ such that

$$
\left((I-K) \boldsymbol{u}_{N},(I-K) \boldsymbol{v}\right)_{k+1}+\langle\lambda, \rho \boldsymbol{v}\rangle_{N, n}=0
$$

and (6) holds.

The uniqueness of $\lambda$ is immediate. 


\section{Convergence Result}

Assume that $f \in H^{k+1}\left((0,1) ; \mathbb{R}^{n}\right)$ and $k \in H^{k}\left((0,1) \times(0,1) ; \mathbb{R}^{n, n}\right)$, then there exists a unique solution $x \in H^{k+1}\left((0,1) ; \mathbb{R}^{n}\right)$ of (1). Moreover, the following convergence result is verified.

Theorem 4. Suppose given $f \in H^{k+1}\left((0,1) ; \mathbb{R}^{n}\right)$ and $\boldsymbol{k} \in H^{k}\left((0,1) \times(0,1) ; \mathbb{R}^{n, n}\right)$. Let denote $\boldsymbol{x} \in H^{k+1}\left((0,1) ; \mathbb{R}^{n}\right)$ the unique solution of $(1)$ and $\boldsymbol{u}_{N} \in \boldsymbol{H}_{N}$ the unique solution of $(4)$. Suppose that the hypothesis (2) holds, where $h$ is mentioned. Then, one has

$$
\lim _{h \rightarrow 0}\left\|\boldsymbol{u}_{N}-\boldsymbol{x}\right\|_{k}=0
$$

Proof. Let $s_{x, T_{N}}$ be the interpolating radial basis function of $x$ on $T_{N}$ from the Wendland function $\phi_{1, k}$, then $s_{x, T_{N}} \in S_{N}$. Thus $J\left(u_{N}\right) \leq J\left(s_{x, T_{N}}\right)$, that also implies that

$$
\left|(I-K) \boldsymbol{u}_{N}\right|_{k+1} \leq\left|(I-K) \boldsymbol{s}_{\boldsymbol{x}, \boldsymbol{T}_{N}}\right|_{k+1} \text {. }
$$

In this case, we have

$$
\left[\left[(I-K) \boldsymbol{u}_{N}\right]\right] \leq\left[\left[(I-K) \boldsymbol{s}_{\boldsymbol{x}, \boldsymbol{T}_{N}}\right]\right]
$$

From this, and that the operator $(I-K)$ is linear and compact in the finite-dimensional space $S_{N}$, and thus bijective, we can deduce that there exists $C_{1}>0$ verifying

$$
\left\|\boldsymbol{u}_{N}\right\|_{k+1} \leq C_{1}\left\|s_{x, T_{N}}\right\|_{k+1}
$$

Taking into account (3), it is verified that there exists $C_{2}>0$ such

$$
\left\|s_{x, T_{N}}\right\|_{k+1} \leq C_{2}\|x\|_{k+1}
$$

and, from here and (8) we obtain that there exists $C>0$ such that

$$
\left\|u_{N}\right\|_{k+1} \leq C\|x\|_{k+1}
$$

Thus, the family $\left(\boldsymbol{u}_{N}\right)_{N \in \mathbb{N}}$ is bounded in $H^{k+1}\left((0,1) ; \mathbb{R}^{n}\right)$, and consequently there exists a sequence $\left(\boldsymbol{u}_{N_{\ell}}\right)_{\ell \in \mathbb{N}}$ extracted from this family, and an element $x^{*} \in H^{k+1}\left((0,1) ; \mathbb{R}^{n}\right)$ such that

$$
x^{*}=\lim _{\ell \rightarrow+\infty} \boldsymbol{u}_{N_{\ell}} \text { weakly in } H^{k+1}\left((0,1) ; \mathbb{R}^{n}\right) .
$$

Suppose that $x^{*} \neq x$; then, from the continuous injection of $H^{k+1}\left((0,1) ; \mathbb{R}^{n}\right)$ into $C\left([0,1] ; \mathbb{R}^{n}\right)$, there exists $\gamma>0$ and a nonempty interval $\omega \subset[0,1]$ such that

$$
\forall t \in \omega, \quad\left\langle x^{*}-x\right\rangle_{n}>\gamma
$$

As this injection is compact, from (9)

$$
\exists \ell_{0} \in \mathbb{N}, \forall \ell \geq \ell_{0},\left\langle\boldsymbol{u}_{N_{\ell}}(t)-x^{*}(t)\right\rangle_{n} \leq \frac{\gamma}{2}
$$

Thus, for any $\ell \geq \ell_{0}$ and $t \in \omega$ it is verified

$$
\left\langle\boldsymbol{u}_{N_{\ell}}(t)-\boldsymbol{x}(t)\right\rangle_{n} \geq\left\langle\boldsymbol{x}^{*}(t)-\boldsymbol{x}(t)\right\rangle_{n}-\left\langle\boldsymbol{u}_{N_{\ell}}(t)-\boldsymbol{x}^{*}(t)\right\rangle_{n}>\frac{\gamma}{2} .
$$

On the other hand, as we are taking $h \rightarrow 0$ along the whole process, using the density condition (2) we can assure that there exists $\ell \in \mathbb{N}$ and $t_{\ell}^{*} \in \omega$ such that $t_{\ell}^{*} \in T_{N_{\ell}} \cap \omega$ and thus

$$
(I-K) \boldsymbol{u}_{N_{\ell}}\left(t_{\ell}^{*}\right)=(I-K) \boldsymbol{x}\left(t_{\ell}^{*}\right)
$$


The operator $I-K$, considering the hypotheses taken from the beginning, it is also a bijection in $C\left((0,1) ; \mathbb{R}^{n}\right)$, and thus $\boldsymbol{u}_{N_{\ell}}\left(t_{\ell}^{*}\right)=x\left(t_{\ell}^{*}\right)$, which is a contradiction with (10). Thus $x^{*}=x$.

For any $\ell \in \mathbb{N}$ it is verified

$$
\left\|\boldsymbol{u}_{N_{\ell}}-\boldsymbol{x}\right\|_{k}^{2}=\left\|\boldsymbol{u}_{N_{\ell}}\right\|_{k}^{2}+\|x\|_{k}^{2}-2\left(\boldsymbol{u}_{N_{\ell}}, x\right)_{k}
$$

Then, from (9) and the compact inclusion of $H^{k+1}\left((0,1) ; \mathbb{R}^{n}\right)$ into $H^{k}\left((0,1) ; \mathbb{R}^{n}\right)$ (see for example [48]), one has

$$
\lim _{\ell \rightarrow+\infty}\left\|\boldsymbol{u}_{N_{\ell}}-\boldsymbol{x}\right\|_{k}=0
$$

Suppose now that $\left\|\boldsymbol{u}_{N}-\boldsymbol{x}\right\|_{k}$ does not tend to 0 as $h$ tends to 0 ; in this case, it would exist $\alpha>0$, and a sequence $\left(\boldsymbol{u}_{N_{\ell}^{\prime}}\right)_{\ell \in \mathbb{N}}$ such that

$$
\forall \ell \in \mathbb{N},\left\|u_{N_{\ell}^{\prime}}-x\right\|_{k}>\alpha
$$

However, the sequence $\left(\boldsymbol{u}_{N_{\ell}^{\prime}}\right)_{\ell \in \mathbb{N}}$ is bounded in $H^{k+1}\left((0,1) ; \mathbb{R}^{n}\right)$ and then, by reasoning as above, we deduce that from this sequence we can extract a subsequence convergent to $x$ in $H^{k}\left((0,1) ; \mathbb{R}^{n}\right)$, what contradicts (12). Thus

$$
\lim _{h \rightarrow 0}\left\|\boldsymbol{u}_{N}-\boldsymbol{x}\right\|_{k}=0
$$

Corollary 1. Under the conditions of Theorem 4 one has

$$
\lim _{h \rightarrow 0}\left\|f-(I-K) \boldsymbol{u}_{N}\right\|_{k}=0 .
$$

Proof. From Theorem 4 and the continuity of the operator $I-K$ we have

$$
\lim _{h \rightarrow 0}(I-K) \boldsymbol{u}_{N}=(I-K) \boldsymbol{x}=f \text { in } H^{k}\left((0,1) ; \mathrm{R}^{n}\right) .
$$

Then, from here the result is obtained.

\section{Computation}

Let us compute the unique solution of (6). The solution of problem (5) can be expressed by

$$
\boldsymbol{u}_{N}=\sum_{i=1}^{N} \boldsymbol{\alpha}_{i} \phi_{1, k}\left(\left|\cdot-t_{i}\right|\right),
$$

with $\alpha_{1}, \ldots, \alpha_{N} \in \mathbb{R}^{n}$.

Consider the basis $\left\{\boldsymbol{B}_{1}, \ldots, \boldsymbol{B}_{N n}\right\}$ of the space $\boldsymbol{S}_{N}$ given, for $\ell=1, \ldots N n$, by

$$
\boldsymbol{B}_{\ell}(t)=\phi_{1, k}\left(\left|t-t_{i}\right|\right) \boldsymbol{e}_{j}
$$

being $i=$ quotient $(\ell-1, n)+1$ and $j=\ell-(i-1) n$.

Then, the solution of (5) can be expressed by

$$
\boldsymbol{u}_{N}=\sum_{\ell=1}^{N n} \alpha_{\ell} \boldsymbol{B}_{\ell},
$$

with $\alpha_{1}, \ldots, \alpha_{N n} \in \mathbb{R}$. 
By replacing in (6), we have

$$
\sum_{\ell=1}^{N n} \alpha_{\ell}\left((I-K) \boldsymbol{B}_{\ell},(I-K) \boldsymbol{v}\right)_{k+1}+\langle\lambda, \rho \boldsymbol{v}\rangle_{N, n}=0, \forall \boldsymbol{v} \in S_{N},
$$

subject to the restrictions

$$
\sum_{\ell=1}^{N n} \alpha_{\ell}(I-K) \boldsymbol{B}_{\ell}\left(t_{i}\right)=f\left(t_{i}\right), \quad i=1, \ldots, N
$$

Taking $\boldsymbol{v}=\boldsymbol{B}_{j}$, for $j=1, \ldots, N n$, we obtain a linear system of order $2 N n$ with unknowns $\alpha_{1}, \ldots, \alpha_{N n}, \lambda_{1}, \ldots, \lambda_{N n} \in \mathbb{R}$, that can be expressed in matrix form as follows:

$$
\left(\begin{array}{ll}
\mathcal{C} & \mathcal{D} \\
\mathcal{D}^{\top} & 0
\end{array}\right)\left(\begin{array}{l}
\boldsymbol{\alpha} \\
\lambda
\end{array}\right)=\left(\begin{array}{l}
\mathbf{0} \\
\boldsymbol{F}
\end{array}\right)
$$

with

$$
\begin{aligned}
& \mathcal{C}=\left(\left((I-K) \boldsymbol{B}_{\ell},(I-K) \boldsymbol{B}_{j}\right)_{k+1}\right)_{\substack{1 \leq \ell \leq N n \\
1 \leq j \leq N n}}, \\
& \mathcal{D}=\left(d_{i j}\right)_{\substack{1 \leq i \leq N n \\
1 \leq j \leq N n}}, \quad \lambda=\left(\lambda_{1}, \ldots, \lambda_{N n}\right)^{\top}, \\
& \boldsymbol{\alpha}=\left(\alpha_{1}, \ldots, \alpha_{N n}\right)^{\top}, \quad \lambda \\
& \boldsymbol{F}=\left(f_{i}\right)_{1 \leq i \leq N n,},
\end{aligned}
$$

being, for $i=1 \ldots, N n$ and $j=1, \ldots, N n$,

$$
d_{i j}=\Pi_{\ell}\left((I-K) \boldsymbol{B}_{r}\left(t_{s}\right)\right),
$$

with $r=$ quotient $(i-1, n)+1, s=q u o t i e n t(j-1, n)+1, \ell=j-(s-1) n$ and for $i=$ $1, \ldots, N n$,

$$
f_{i}=\Pi_{\ell}\left(f\left(t_{s}\right)\right),
$$

with $s=$ quotient $(i-1, n)$ and $\ell=i-(s-1) n$.

\section{Numerical Examples}

To check the validity of the described method for approximating the solution of Problem (1) we present some numerical experiments.

In order to show the accuracy of the method, we have computed two relative error estimations, given by the expressions

$$
E_{1}=\frac{1}{1000} \sum_{i=1}^{1000}\left\langle\boldsymbol{f}\left(a_{i}\right)-(I-K) \boldsymbol{u}_{N}\left(a_{i}\right)\right\rangle_{n}
$$

which estimates how close $\boldsymbol{u}_{N}$ is to the solution of (1) and

$$
E_{2}=\sqrt{\frac{\sum_{i=1}^{1000}\left\langle\boldsymbol{u}_{N}\left(a_{i}\right)-\boldsymbol{x}\left(a_{i}\right)\right\rangle_{n}^{2}}{\sum_{i=1}^{1000}\left\langle\boldsymbol{x}\left(a_{i}\right)\right\rangle_{n}^{2}}},
$$

which is an approximation of the relative error of $\boldsymbol{u}_{N}$ with respect to $x$ in $L^{2}\left((0,1) ; \mathrm{R}^{n}\right)$ being $\left\{a_{1}, \ldots, a_{1000}\right\} \subset[0,1]$ thousand distinct random points.

From Theorem 4 and Corollary 1 , these relative error estimations $E_{1}$ and $E_{2}$ tend to 0 as $h$ tends to 0 . 
Moreover, in all the examples, the discrete space that we use to calculate the approximated solution $\boldsymbol{u}_{N}$ is the radial basis function space constructed from the Wendland function $\phi_{1,1}$ and the centers set $\boldsymbol{T}_{N}=\left\{t_{i}=\frac{i}{N}, i=0, \ldots, N\right\}$.

In order to compute the numerical integrals, we have used the following quadrature formula (see [52])

$$
\begin{aligned}
\int_{a}^{b} g(t) d t \approx & \sum_{i=6}^{n-3} g\left(\xi_{i}\right)+h\left(\frac{206}{1575}\left(g\left(\xi_{1}\right)+g\left(\xi_{n+2}\right)\right)+\frac{107}{128}\left(g\left(\xi_{2}\right)+g\left(\xi_{n+1}\right)\right)+\right. \\
& \frac{6019}{5760}\left(g\left(\xi_{3}\right)+g\left(\xi_{n}\right)\right)+\frac{9467}{9600}\left(g\left(\xi_{4}\right)+g\left(\xi_{n-1}\right)\right)+ \\
& \left.\frac{13,469}{13,440}\left(g\left(\xi_{5}\right)+g\left(\xi_{n-2}\right)\right)\right)
\end{aligned}
$$

where $h=\frac{b-a}{n}$ and

$$
\xi_{1}=a, \quad \xi_{n+2}=b, \quad \xi_{i}=a+\frac{2 i-1}{2} h, i=2, \ldots, n+1 .
$$

This formula has an error order of $O\left(h^{6}\right)$ for $g \in C^{6}([a, b])$.

Example 1. We consider the following Volterra equation system of order 2

$$
\left\{\begin{array}{l}
x_{1}(t)-\int_{0}^{t}\left((t-s)^{3} x_{1}(s)+(t-s)^{2} x_{2}(s)\right) d s=t-\frac{t^{5}}{12} \\
x_{2}(t)-\int_{0}^{t}\left((t-s)^{4} x_{1}(s)+(t-s)^{3} x_{2}(s)\right) d s=t^{2}-\frac{t^{6}}{20} .
\end{array}\right.
$$

The exact solution is

$$
x_{1}(t)=t, \quad x_{2}(t)=t^{2} .
$$

Table 2 shows the relative error estimations for distinct values of $N$.

Table 2. Computed relative error estimations for Example 1 from some values of $N$.

\begin{tabular}{ccc}
\hline $\boldsymbol{N}$ & $\boldsymbol{E}_{\mathbf{1}}$ & $\boldsymbol{E}_{\mathbf{2}}$ \\
\hline 5 & $2.1868 \times 10^{-2}$ & $3.1058 \times 10^{-2}$ \\
10 & $3.6034 \times 10^{-3}$ & $4.8048 \times 10^{-3}$ \\
20 & $6.2683 \times 10^{-4}$ & $8.2990 \times 10^{-4}$ \\
30 & $2.0727 \times 10^{-4}$ & $3.0254 \times 10^{-4}$ \\
40 & $1.0215 \times 10^{-4}$ & $1.2509 \times 10^{-4}$ \\
50 & $6.4520 \times 10^{-5}$ & $9.2824 \times 10^{-5}$ \\
\hline
\end{tabular}

Example 2. We consider the following Volterra equation system of order 2

$$
\left\{\begin{array}{l}
x_{1}(t)-\int_{0}^{t}\left(e^{t-s} x_{1}(s)+e^{t+s} x_{2}(s)\right) d s=e^{t}(1-2 t), \\
x_{2}(t)-\int_{0}^{t}\left(-e^{t-s} x_{1}(s)+e^{t+s} x_{2}(s)\right) d s=e^{-t} .
\end{array}\right.
$$

The exact solution is

$$
x_{1}(t)=e^{t}, \quad x_{2}(t)=e^{-t} .
$$

Table 3 shows the relative error estimations for distinct values of $N$. 
Table 3. Computed relative error estimations for Example 2 from some values of $N$.

\begin{tabular}{ccl}
\hline $\boldsymbol{N}$ & $\boldsymbol{E}_{\mathbf{1}}$ & $\boldsymbol{E}_{\mathbf{2}}$ \\
\hline 5 & $3.0586 \times 10^{-2}$ & $2.5854 \times 10^{-2}$ \\
10 & $6.4473 \times 10^{-3}$ & $3.7229 \times 10^{-3}$ \\
20 & $1.1610 \times 10^{-3}$ & $6.3689 \times 10^{-4}$ \\
30 & $4.4048 \times 10^{-4}$ & $2.2905 \times 10^{-4}$ \\
40 & $1.5159 \times 10^{-4}$ & $1.1068 \times 10^{-4}$ \\
50 & $9.9079 \times 10^{-5}$ & $6.3629 \times 10^{-5}$ \\
\hline
\end{tabular}

Example 3. We consider the following Volterra equation system of order 3

$$
\left\{\begin{array}{l}
x_{1}(t)-\int_{0}^{t}\left(x_{1}(s)+t x_{3}(s)\right) d s=-t+t^{2} \\
x_{2}(t)-\int_{0}^{t}\left((t+s) x_{1}(s)+x_{2}(s)+(t-s) x_{3}(s)\right) d s=1-t-\frac{t^{4}}{2} \\
x_{3}(t)-\int_{0}^{t}\left((-t-s) x_{1}(s)-x_{2}(s)+(-t+s) x_{3}(s)\right) d s=-t-t^{2}+\frac{t^{4}}{2} .
\end{array}\right.
$$

The exact solution is

$$
x_{1}(t)=t^{2}, \quad x_{2}(t)=1, \quad x_{3}(t)=-t^{2} .
$$

Table 4 shows the relative error estimations for distinct values of $N$.

Table 4. Computed relative error estimations for Example 3 from some values of $N$.

\begin{tabular}{ccc}
\hline$N$ & $E_{1}$ & $E_{2}$ \\
\hline 5 & $2.0024 \times 10^{-2}$ & $3.5705 \times 10^{-2}$ \\
10 & $2.4457 \times 10^{-3}$ & $5.2296 \times 10^{-3}$ \\
20 & $2.9878 \times 10^{-4}$ & $7.5222 \times 10^{-4}$ \\
30 & $7.5462 \times 10^{-5}$ & $2.6518 \times 10^{-4}$ \\
40 & $2.6133 \times 10^{-5}$ & $1.1834 \times 10^{-4}$ \\
50 & $1.0932 \times 10^{-5}$ & $7.1453 \times 10^{-5}$ \\
\hline
\end{tabular}

\section{Conclusions}

We conclude that the above presented experiments (see Tables 1-4)) confirm the validity of the method and justify the convergence results given in Theorem 4 and Corollary 1 . In fact, in all our experiments (see the Examples 1-3), by using small values of $N$, we obtain a significant good order of approximation using the relative errors $E_{1}$ and $E_{2}$ considered. So, our original goal to devise an appropriate variational procedure that is capable of solving this type of problems in a precise and efficient way has been completely accomplished.

As compared with the other recently published works, for example [36-38,40,41], they do not study convergence results. Likewise, our technique gives an acceptable accuracy with a small use of data, resulting also a low computational cost.

In ([37], Tables 1 and 2) the mean of the error is of the order $10^{-5}$. We have obtained the same order of error with only 50 points.

In [40] the authors use Bernstein polynomials and the degree of its approximation is of order $10^{-4}$ in most of the tables. The same happens in ([41], Table 4), it uses the simple block-by-block method and its degree of approximation is about $10^{-3}$.

In order to do more research on this topic in the future, among some of the open problems that we consider are: 
- a numerical comparison between our method and many others in the literature,

- the theoretical study of the order of convergence of the presented method,

- the adaptation of this procedure to find the numerical solution of the linear systems of 2D Volterra integral equations of the second kind.

Author Contributions: All authors contributed equally to formal analysis, investigation, methodology, project administration, writing - original draft and writing-review \& editing. All authors have read and agreed to the published version of the manuscript.

Funding: This work was supported by FEDER/Junta de Andalucía-Consejería de Transformación Económica, Industria, Conocimiento y Universidades (Research Project A-FQM-76-UGR20, University of Granada) and by the Junta de Andalucía (Research Group FQM191).

Institutional Review Board Statement: Not applicable.

Informed Consent Statement: Not applicable.

Data Availability Statement: Not applicable.

Acknowledgments: We acknowledge the anonymous referees for their useful comments and suggestions, as well as the Department of Applied Mathematics of the University of Granada and the Mathematics Journal Editorial Board, for the financial aid offered for the final cost of the APC.

Conflicts of Interest: The authors declare no conflict of interest.

\section{References}

1. Volterra, V. Leçons sur les équations intégrales et intégro-différentielles; Gauthier-Villars: Paris, France, 1913.

2. Corduneanu, C.; Sandberg, I.W. Volterra Equations and Applications; Gordon and Breach: Amsterdam, The Netherlands, 2000.

3. Baker, C.T.H. Numerical Analysis of Volterra Functional and Integral Equations. In The State of the Art in Numerical Analysis; Duff, I.S., Watson, G.A., Eds.; Oxford University Press: New York, NY, USA, 1997; pp. 193-222.

4. Cahlon, B. Numerical solution of nonlinear Volterra integral equations. J. Comput. Appl. Math. 1981, 7, 121-128. [CrossRef]

5. Shampine, L.F. Solving Volterra integral equations with ODE codes. IMA J. Numer. Anal. 1988, 8 ,37-41. [CrossRef]

6. Brunner, H. 1896-1996: One hundred years of Volterra integral equations of the first kind. Appl. Numer. Math. 1997, 24, 83-93. [CrossRef]

7. Brunner, H. ; Pedas, A.; Vainikko, G. The piecewise polynomial collocation method for nonlinear weakly singular Volterra equations. Math. Comp. 1999, 68, 1079-1095. [CrossRef]

8. Elnagar, G.N.; Kazemi, M. Chebyshev spectral solution of nonlinear Volterra-Hammerstein integral equations. J. Comput. Appl. Math. 1996, 76, 147-158. [CrossRef]

9. Daubechies I. Ten Lectures on Wavelets; CBMS-NSF Regional Conference Series in Applied Mathematics; SIAM: Philadelphia, PA, USA, 1992.

10. Aziz, I.; Siraj-ul-Islam. New algorithms for the numerical solution of nonlinear Fredholm and Volterra integral equations using Haar wavelets. J. Comput. Appl. Math. 2013, 239, 333-345. [CrossRef]

11. Babolian, E.; Shahsavaran, A. Numerical solution of nonlinear Fredholm integral equations of the second kind using Haar wavelets. J. Comput. Appl. Math. 2009, 225, 87-95. [CrossRef]

12. Lepik, Ü. Solving integral and differential equations by the aid of non-uniform Haar wavelets. Appl. Math. Comput. 2008, 198, 326-332. [CrossRef]

13. Maleknejad, K.; Mirzaee, F. Using rationalized Haar wavelet for solving linear integral equations. Appl. Math. Comput. 2005, 160, 579-587. [CrossRef]

14. Mirzaae, F. Numerical computational solution of the linear Volterra integral equations systems via rationalized Haar functions. J. King Saud Univ. Sci. 2010, 22, 265-268. [CrossRef]

15. Maleknejad, K.; Almasieh, H.; Roodaki, M. Triangular functions (TF) method for the solution of nonlinear Volterra-Fredholm integral equations. Comm. Nonlinear Sci. Numer. Simulat. 2010, 15, 3293-3298. [CrossRef]

16. Deb, A.; Sarkar, G.; Sengupta, A. Triangular Orthogonal Functions for the Analysis of Continuous Time Systems; Elsevier: New Delhi, India, 2007.

17. Roodaki, M.; Almasieh, H. Delta basis functions and their applications to systems of integral equations. Comput. Math. Appl. 2012, 63, 100-109. [CrossRef]

18. Ghasemi, M.; Tavassoli Kajani, M.; Babolian, E. Numerical Solutions of the Nonlinear Volterra-Fredholm Integral Equations by Using Homotopy Perturbation Method. Appl. Math. Comput. 2007, 188, 446-449. [CrossRef]

19. Wazwaz, A.-M. The combined Laplace transform-Adomian decomposition method for handling nonlinear Volterra integrodifferential equations. Appl. Math. Comput. 2010, 216, 1304-1309. [CrossRef]

20. Yousefi, S.; Razzaghi, M. Legendre wavelets method for the nonlinear Volterra-Fredholm integral equations. Math. Comput. Simulat. 2005, 70, 1-8. [CrossRef] 
21. Maleknejad, K.; Kajani, M.T. Solving integro-differential equation by using Hybrid Legendre and block-pulse functions. Int. J. Appl. Math. 2002, 11, 67-76.

22. El-Borai, M.; Abdou, M.A.; Badr, A.A.; Basseem, M. Chebyshev Polynomials and Fredholm-Volterra integral equation. Int. J. Appl. Math. Mech. 2008, 4, 78-92.

23. Youssri, Y.H.; Hafez, R. M. Chebyshev collocation treatment of Volterra-Fredholm integral equation with error analysis. Arab. J. Math. 2020, 9, 471-480. [CrossRef]

24. Berenguer, M.I.; Gámez, D. ; Garralda-Guillém, A.I. ; Ruiz Galán, M. ; Serrano Pérez, M.C. Analytical Techniques for a Numerical Solution of the Linear Volterra Integral Equation of the Second Kind. Abstr. Appl. Anal. 2009, 14936. [CrossRef]

25. Berenguer, M.I.; Gámez, D. ; Garralda-Guillém, A.I. ; Ruiz Galán, M. ; Serrano Pérez, M.C. Nonlinear Volterra Integral Equation of the Second Kind and Biorthogonal Systems. Abstr. Appl. Anal. 2010, 135216. [CrossRef]

26. Şahn, N.; Yüzbaşi, S..; Glsu, M. A collocation approach for solving systems of linear Volterra integral equations with variable coefficients. Comput. Math. Appl. 2011, 62, 755-769. [CrossRef]

27. Balakumar, V.; Murugesan, K. Numerical solution of Volterra integral-algebraic equations using block pulse functions. Appl. Math. Comput. 2015, 263, 165-170. [CrossRef]

28. Yang, L.-H.; Shen, J.-H.; Wang, Y. The reproducing kernel method for solving the system of the linear Volterra integral equations with variable coefficients. J. Comput. Appl. Math. 2012, 236, 2398-2405. [CrossRef]

29. Delves, L.M.; Mohamed, J.L. Computational Methods for Integral Equations; Cambridge University Press: New York, USA, 1985.

30. Van der Houwen, P.J.; Sommeijer, B.P. Euler-Chebyshev methods for integro-differential equations. Appl. Numer. Math. 1997, 24, 203-218. [CrossRef]

31. Draidi, W.; Qatanani, N. Numerical Schemes for solving Volterra integral equations with Carleman kernel. Int. J. Appl. Math. 2005, 31, 647-669. [CrossRef]

32. Issa, A.; Qatanani, N.; Daraghmeh, A. Approximation Techniques for Solving Linear Systems of Volterra Integro-Differential Equations. J. Appl. Math. 2020, 1-12. [CrossRef]

33. Aggarwal, S.; Gupta, A.R. Solution of Linear Volterra Integro-Differential Equations of Second Kind Using Kamal Transform J. Emerg. Technol. Innov. Res. 2019, 6, 741-747.

34. Chauhan, R.; Aggarwal, S. Laplace Transform for Convolution Type Linear Volterra Integral Equation of Second Kind. J. Adv. Res. Appl. Math. Stat. 2019, 4, 1-7.

35. Mahgoub, M.M.A. The new integral transform: Sawi transform. Adv. Theor. Appl. Math. 2019, 14, 81-87.

36. Islam, M.S.; Islam, M.S.; Bangalee, M.Z.I.; Khan, A.K.; Halder, A. Approximate Solution of Systems of Volterra Integral Equations of Second Kind by Adomian Decomposition Method. Dhaka. Univ. J. Sci. 2015 63, 15-18. [CrossRef]

37. Hong, Z.; Fang, X.; Yan, Z., Hao, H. On Solving a System of Volterra Integral Equations with Relaxed Monte Carlo Method. J. Appl. Math. Phys. 2016, 4, 1315-1320. [CrossRef]

38. Khan, F.; Omar, M.; Ullah, Z. Discretization method for the numerical solution of 2D Volterra integral equation based on two-dimensional Bernstein polynomials. AIP Adv. 2018, 8, 125209. [CrossRef]

39. Aggarwal, S.; Sharma, N.; Chauhan, R. Solution of Linear Volterra Integral Equations of Second Kind Using Mohand Transform. IJRAT 2018, 6, 3098-3102.

40. Muhammad, A.M.; Ayal, A.M. Numerical Solution of Linear Volterra Integral Equation with Delay using Bernstein Polynomial. IEJME 2019, 14, 735-740. [CrossRef]

41. Kasumo, C. On the Approximate Solutions of Linear Volterra Integral Equations of the First Kind. Appl. Math. Sci. 2020, 14, 141-153. [CrossRef]

42. Bjornsson, H.; Hafstein, S. Advanced algorithm for interpolation with Wendland functions. In Informatics in Control, Automation and Robotics (ICINCO 2019); Lecture Notes in Electrical Engineering; Gusikhin O., Madani K., Zaytoon J., Eds.; Springer: New York, NY, USA, 2019; pp. 99-117.

43. Assari, P.; Dehghan, M. A meshless local Galerkin method for solving Volterra integral equations deduced from nonlinear fractional differential equations using the moving least squares technique. Appl. Numer. Math. 2019, 143, 276-299. [CrossRef]

44. Farshadmoghadam, F.; Azodi, H.D. ; Yaghouti, M.R. An improved radial basis functions method for the high-order VolterraFredholm integro-differential equations. Math. Sci. 2021. [CrossRef]

45. Maleknejad, K.; Mohammadikia, H.; Rashidinia, J. A numerical method for solving a system of Volterra-Fredholm integral equations of the second kind based on the meshless method. Afrika Mat. 2018, 29, 955-965. [CrossRef]

46. Uddin, M.; Ullah, N.; Ali-Shah, S.I. RBF Based Localized Method for Solving Nonlinear Partial Integro-Differential Equations. Comput. Model. Eng. Sci. 2020, 123, 957-972. [CrossRef]

47. Zhang, H.; Chen, Y.; Nie, X. Solving the Linear Integral Equations Based on Radial Basis Function Interpolation. J. Appl. Math. 2014, 1-18. Available online: https://www.hindawi.com/journals/jam/2014/793582/ (accessed on 20 October 2021). [CrossRef]

48. Atkinson, K.; Weiman, H. Theoretical Numerical Analysis. A Functional Analysis Framework, 2nd ed.; Springer: New York, NY, USA, 2005

49. Kouibia, A.; Pasadas, M.; Rodriguez, M.L. A variational method for solving Fredholm integral systems. Appl. Numer. Math. 2012, 66, 1041-1049. [CrossRef]

50. Wendland, H. Error Estimates for Interpolation by Compactly Supported Radial Basis Functions of Minimal Degree. J. Approx. Theory 1998, 93, 258-272. [CrossRef] 
51. Wendland, H. Meshless Garlekin Methods Using Radial Basis Functions. Math. Comp. 1999, 228, 1521-1531. [CrossRef]

52. Sablonière, P. Univariate spline quasi-interpolants and applications to numerical analysis. Rend. Sem. Mat. Univ. Pol. Torino 2005, 63, 107-118. 